\title{
True Arterial Stiffness Does Not Change between Dialysis Sessions during 1 Week in Outpatients on Intermitted Hemodialysis
}

\author{
Alexander Reshetnik ${ }^{a} \quad$ Daniel Wrobel $^{b} \quad$ Georg Wirtz $^{b} \quad$ Markus Tölle $^{a}$ \\ Kai-Uwe Eckardt ${ }^{a}$ Markus van der Giet ${ }^{a}$ \\ ${ }^{a}$ Charité, Universitätsmedizin Berlin, A Corporate Member of Freie Universität Berlin, \\ Humboldt-Universität zu Berlin, Department of Nephrology and Intensive Care Medicine, \\ Berlin Institute of Health, Berlin, Germany; ${ }^{b}$ Diavital Dialysis Centre, Kamen, Germany
}

\section{Keywords}

Arterial stiffness $\cdot$ Pulse wave velocity $\cdot$ Blood pressure dependence $\cdot$ Dialysis · End-stage renal disease

\begin{abstract}
Introduction: End-stage renal disease (ESRD) is associated with exponentially elevated cardiovascular mortality. Arterial stiffness (AS) - usually expressed with pulse wave velocity $(P W V)$ - is an established independent predictor of cardiovascular risk beyond the traditional risk factors. Higher PWV values are frequently observed in patients with ESRD. Due to the intrinsic physiologic relationship between PWV and prevailing arterial pressure, PWV can change without relevant changes in the arterial wall structure, and thus an individual pressureindependent expression of PWV is essential. Methods: The study is a single-center observational study. Repeated measurements of blood pressure (BP) and pulse wave analysis were performed during each dialysis session of 1 week. Aortic PWV was then adjusted to $120 \mathrm{~mm}$ $\mathrm{Hg}$ central systolic BP (PWV120) based on individually determined relationship. PWV120 values were compared between single sessions. Calculation of the PWV120 was performed retrospectively. Results: Fifty-four subjects were included, $61.1 \%$ of whom were male. The median age was 75.5 years, and median dialysis vintage was 33.1 months. Mean systolic/ diastolic BP was $121.4 / 70.5 \mathrm{~mm} \mathrm{Hg}$, and the median heart rate was 64.6 beats/min. Mean PWV was $10.9 \mathrm{~m} / \mathrm{s}$, and mean PWV120 was $11.3 \mathrm{~m} / \mathrm{s}$. PWV120 did not change across single dialysis session during 1 week, while systolic, diastolic BP, PWV, and ultrafiltration volume differed
\end{abstract}


significantly. Discussion/Conclusions: Our data suggest that true AS does not change in the short-term course in dialysis patients. The observed changes in PWV are rather associated with BP change due to intrinsic pressure dependence. Our analytical approach represents a novel method for this purpose, which is easy in performance and also applicable for large interventional trials and clinical practice.

(c) 2019 The Author(s)

Published by S. Karger AG, Basel

\section{Introduction}

Arterial stiffness (AS) reflects the damage of the arterial vessel wall [1]. Its association with increased cardiovascular morbidity and mortality has been shown in different populations [2, 3]. Chronic kidney disease (CKD) has been associated with increased AS [4]. The cardiovascular disease burden is disproportionally high in patients with CKD and particularly in end-stage renal disease [5]. Thus, assessment of the AS and developing of the treatment strategies to decrease AS in these patients are of significant clinical relevance. However, one of the relevant caveats about AS, which is usually characterized by pulse wave velocity (PWV), is its intrinsic dependence from prevalent blood pressure (BP) [6-8]. As a consequence, change in the BP causes a change in PWV without any change in the arterial wall structure. Specifically, cyclic changes in BP and hydration status in hemodialysis patients could alter PWV values. Whether "true" PWV reflecting the real change in arterial wall structure changes remains unknown.

Available modern oscillometric devices can perform pulse wave analysis and reliably calculate aortic PWV in the clinical routine, which is proofed to be valid compared to tonometric and invasive measurements [9, 10]. Multiple PWV measurements can easily be performed using this technique. Based on the analysis of the multiple PWV measurements in a single patient, we developed a method to obtain an individual BP-independent PWV, which would better reflect the true change in the arterial wall structure. We hypothesized that individual BP-independent PWV would not alter between dialysis sessions in 1 week.

\section{Materials and Methods}

\section{Study Collective}

Adult persons (age $\geq 18$ years) on stable hemodialysis or hemodiafiltration regimen were recruited from the outpatient dialysis unit in Kamen, Germany, from March to April 2017. Exclusion criteria were pregnancy, arterial stenosis proximal or at the measurement site, former dialysis fistula at the measurement site, active infection, arrhythmias making peripheral oscillometric BP measurement, and/or pulse wave analysis impossible. All included subjects performed written informed consent. The Ethical Committee of the medical association Westphalia-Lippe and the University of Muenster, Germany, approved the study. All dialysis procedures were performed with Surdial X dialysis machines (Nipro, Osaka, Japan). Clinical data, including comorbidities, concurrent medication, and laboratory data, were obtained from the electronic and paper-based patient records. We considered patients having hypertension when they have had already a previously established diagnosis of hypertension or have been taking antihypertensive drugs. We considered patients having hyperlipoproteinemia when they have had a previously established diagnosis of hyperlipoproteinemia or have been taking cholesterol-lowering drugs. The study schematic is shown in Figure 1. 
Fig. 1. Study schematic. BP, blood pressure; PWV, pulse wave velocity;

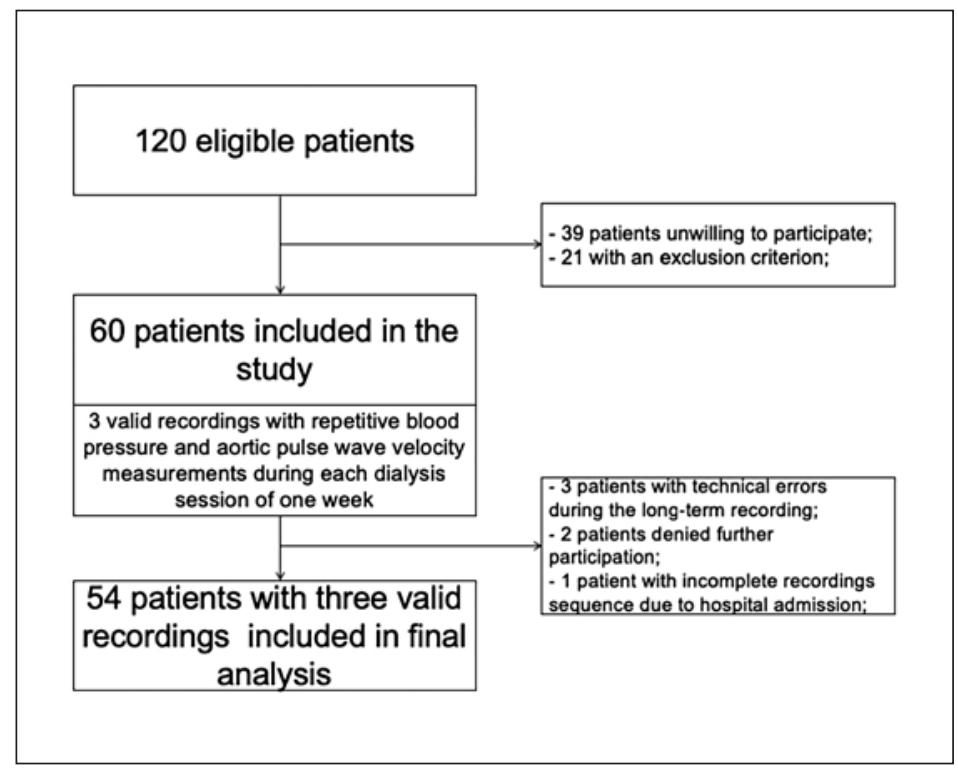

\section{Hemodynamic Measurements}

All BP and pulse wave analysis measurements were performed with Mobil-O-Graph-24 h-pulse wave analysis Monitor (I.E.M. GmbH, Stolberg, Germany), and data analysis was performed with HMS Client Software, version 5.2. Mobil-O-Graph uses ARCSolver method to calculate aortic PWV [11]. Upper arm circumference was measured before the measurement to apply appropriate cuff (Size S: 20-24 cm, M: 25-32 cm, L: 33-38 cm, XL: 39-55 cm). The recordings were performed at the non-fistula arm. The first measurement was started when a patient has taken a comfortable position in the dialysis chair. The device repeated the measurement automatically every $15 \mathrm{~min}$ until the dialysis session ended. Additional on-demand measurements were started manually. Single point measurements of the aortic PWV and central systolic BP were plotted together, and individual change in aortic PWV related to the change in central systolic BP was visualized (online suppl. Figure, see www. karger.com/doi/10.1159/000504138). A linear equation was generated based on the point cloud. The point of interception with Y-axis was defined as PWVbaseline and the slope of the line as PWVslope. Consequently, PWV at 120 mm Hg central systolic BP (PWV120) was calculated as PWVslope $\times 120+$ PWVbaseline. Calculation of the PWVbaseline and PWVslope has been performed retrospectively.

\section{Statistics}

SPSS version 25.0 (IBM, Armonk, NY, USA) was used for the statistical analysis. Normally distributed parameters are reported as mean and SD. Nonnormally distributed parameters are reported as median and interquartile range. Definition of normal distribution was based on the results of the Kolmogorov-Smirnov test. All categorical data were presented as the total number and percentage of the total study population. To describe the hemodynamic values of the single dialysis days in a week, Friedman-test and Friedmans ANOVA post hoc test were used. Hemoglobin A1c values were available for 36 patients. Two-sided $p$ value below 0.05 was considered as statistically significant. 


\section{Kidney \\ Blood Pressure \\ Research}

Table 1. Baseline characteristics of the study collective

\begin{tabular}{l|l}
\hline Kidney Blood Press Res 2020;45:51-60 \\
\hline DOI: 10.1159/000504138 & $\begin{array}{l}\text { @ 2019 The Author(s). Published by S. Karger AG, Basel } \\
\text { www.karger.com/kbr }\end{array}$ \\
\hline
\end{tabular}

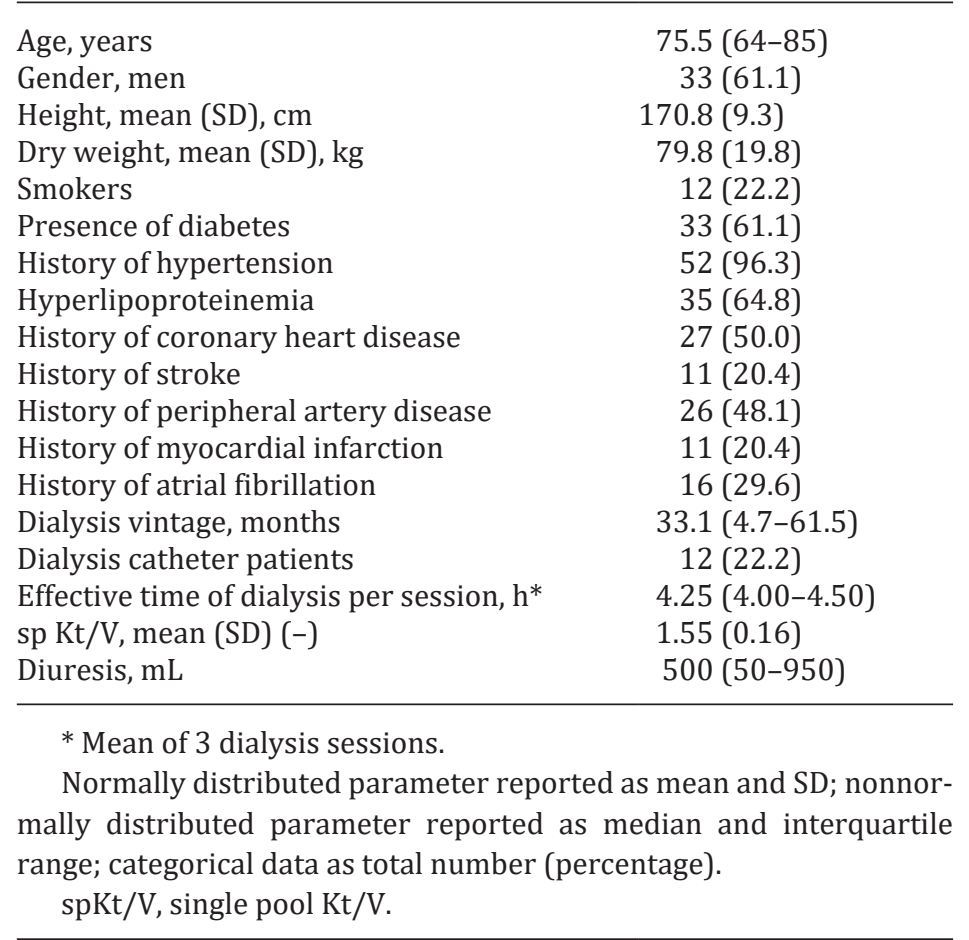

\section{Results}

Fifty-four subjects were included in the analysis, 33 (61.1\%) of whom were male. The median age was 75.5 years, and the mean dry weight of the patients was $79.8 \mathrm{~kg}$. The median dialysis vintage was 33.1 months, and the mean single pool Kt/V was 1.55 . Almost all study participants (96.3\%) had an established diagnosis of arterial hypertension, diabetes mellitus (61.1\%), and hyperlipoproteinemia (64.8\%). About $22.2 \%$ of the patients were dialyzed via an indwelling central venous catheter, and others were dialyzed via a fistula. Baseline characteristics of the study collective are further described in Table 1. People with diabetes were well controlled with the mean hemoglobin A1c of 6.4\%. Median cholesterol level was $157 \mathrm{mg} /$ dL. Almost $76 \%$ of the study collective was on a renin-angiotensin blocker. The same percentage of patients was on a loop diuretic, and the median residual diuresis was $500 \mathrm{~mL} /$ day. In total, $51.9 \%$ took a statin. The detailed information regarding laboratory parameters and comedication is given in Table 2. Mean systolic and diastolic BP of the study population was $121.4 / 70.5 \mathrm{~mm} \mathrm{Hg}$, and the median heart rate was 64.4 beats/min. PWV adjusted to 120 mm Hg central systolic BP was $11.3 \mathrm{~m} / \mathrm{s}$ compared to PWV, which was $10.9 \mathrm{~m} / \mathrm{s}$. Table 3 gives extended information about the hemodynamic parameters of the patients.

Systolic, diastolic, central systolic, central diastolic BP, PWV, and ultrafiltration volume are significantly higher on the first dialysis session during the week compared to second and third dialysis sessions. In contrast, PWVslope, PWVbaseline, and PWV120 did not differ significantly between the dialysis sessions within 1 week (Fig. 2). Univariable linear regression analysis showed diastolic BP, mean arterial BP, age, smoker status, and intact parathyroid hormone (iPTH) as significant impact parameters on PWV120. These parameters were entered in the multiple linear regression analysis, which revealed age as only independent, statistically significant impact parameter on PWV120. The change in PWV120 was not predictable by the change in systolic, diastolic BP, heart rate, and ultrafiltration volume between the dialysis sessions during 1 week (Fig. 3). Table 4 gives a comparison of hemody- 
Kidney

Blood Pressure

Research

Table 2. Relevant laboratory parameters and comedication

\begin{tabular}{l|l}
\hline \multicolumn{2}{l}{ Kidney Blood Press Res 2020;45:51-60 } \\
\hline DOI: $10.1159 / 000504138$ & $\begin{array}{l}\text { (c) 2019 The Author(s). Published by S. Karger AG, Basel } \\
\text { www.karger.com/kbr }\end{array}$ \\
\hline
\end{tabular}

Reshetnik et al.: BP Dependence of AS in ESRD

HbA1c, mean (SD), \%

$6.4(1.3)^{*}$

$\mathrm{Hb}$, mean (SD), g/dL

$10.8(1.1)$

Total protein, $\mathrm{g} / \mathrm{dL}$

$6.6(6.2-6.9)$

Albumin, $\mathrm{g} / \mathrm{dL}$

Cholesterol, mg/dL

$55.5(52.0-59.1)$

157 (126-189)

High-density lipoprotein, mean (SD), mg/dL

$43.9(13.2)$

Low-density lipoprotein, mean (SD), mg/dL

$91.1(36.8)$

iPTH, ng/L

$228(105-424)$

Calcium phosphate product, mean (SD), $\mathrm{mmol}^{2} / \mathrm{L}^{2} \quad 3.6(1.1)$

Renin-angiotensine-system blockade

$41(75.9)$

$28(51.9)$

5 (9.3)

Aldosterone antagonists

$12(22.2)$

Thiazids

Loop diuretics

$41(75.9)$

$40(74.1)$

$3(5.6)$

Alpha blockers

8 (14.8)

5 (9.3)

1 (1.9)

$32(59.3)$

$15(27.8)$

28 (51.9)

Direct vasodilators

Platelet aggregation inhibitors

Anticoagulation

Statins

* Data from 36 patients available.

Normally distributed parameter reported as mean and SD; nonnormally distributed parameter reported as median and interquartile range; categorical data as total number (percentage).

iPTH, intact parathyroid hormone; HbA1c, hemoglobin A1c.

Table 3. Hemodynamic parameters of the study collective
Systolic BP, mean (SD), mm Hg

Diastolic BP, mean (SD), mm Hg

Pulse pressure, $\mathrm{mm} \mathrm{Hg}$

Mean arterial pressure, mean (SD), $\mathrm{mm} \mathrm{Hg}$

Heart rate, $b / \mathrm{min}$

Central systolic BP, mean (SD), mm Hg

Central diastolic BP, $\mathrm{mm} \mathrm{Hg}$

$\mathrm{PWV}, \mathrm{m} / \mathrm{s}$

PWVslope, $\mathrm{m} / \mathrm{s} \times \mathrm{mm} \mathrm{Hg}$

PWVbaseline, $\mathrm{m} / \mathrm{s}$

PWV120, m/s

\author{
$121.4(15.7)$ \\ 70.5 (10.9) \\ $49.3(42.2-56.5)$ \\ $93.8(12.3)$ \\ $64.4(58.6-70.2)$ \\ 108.5 (13.8) \\ $70.1(62.3-78.0)$ \\ $10.9(9.1-12.7)$ \\ $0.0350(0.0322-0.0378)$ \\ $7.1(5.2-9.0)$ \\ $11.3(9.5-13.1)$
}

Parameters reported as mean/median of 3 dialysis sessions during 1 week; PWV120, pulse wave velocity adjusted to the level of $120 \mathrm{~mm}$ $\mathrm{Hg}$ central systolic BP and calculated as PWVbaseline $+120 \mathrm{~mm} \mathrm{Hg} \times$ PWVslope; normally distributed parameter reported as mean and SD; non-normally distributed parameter reported as median and interquartile range.

BP, blood pressure; PWV, pulse wave velocity; PWV120, PWV adjusted to $120 \mathrm{~mm} \mathrm{Hg}$ central systolic BP. 


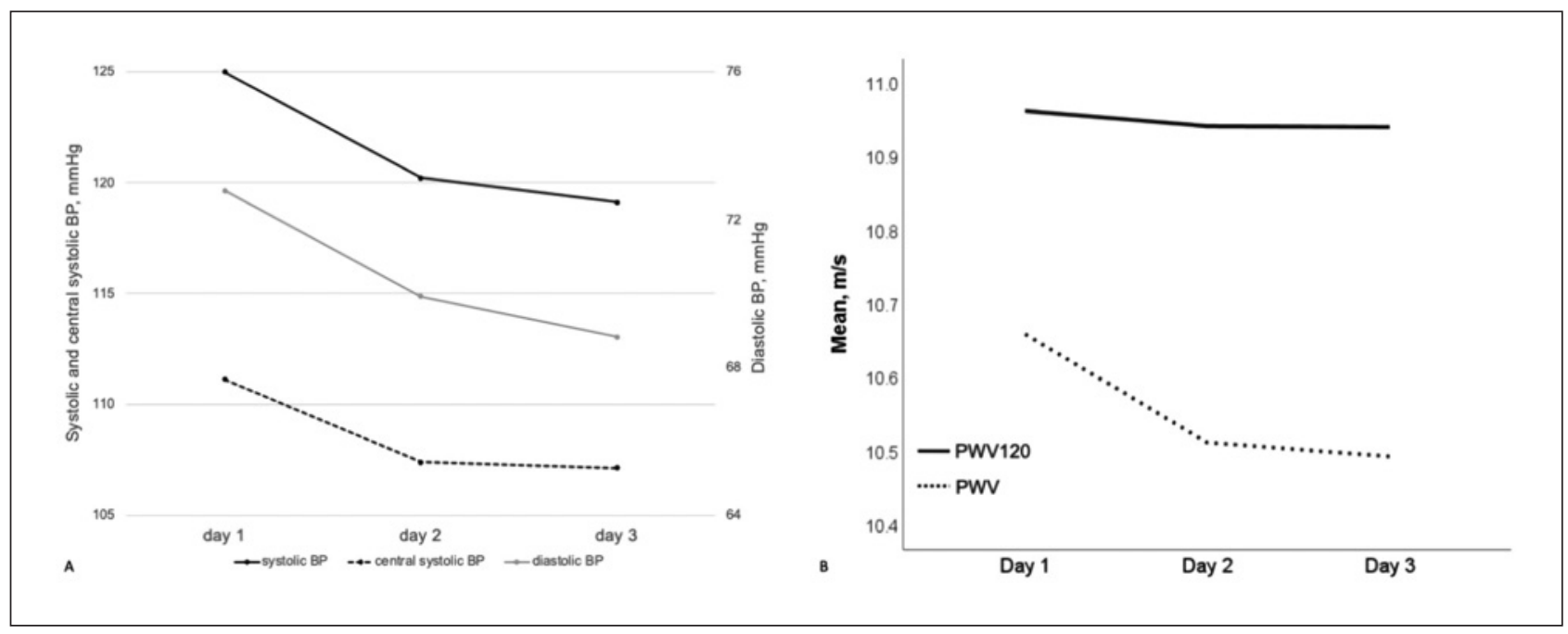

Fig. 2. Change in (A) systolic, diastolic, central systolic BP and (B) PWV and PWV120 between the dialysis days of 1 week. BP, blood pressure; PWV, pulse wave velocity; PWV120, PWV adjusted to $120 \mathrm{~mm} \mathrm{Hg}$ central systolic BP.

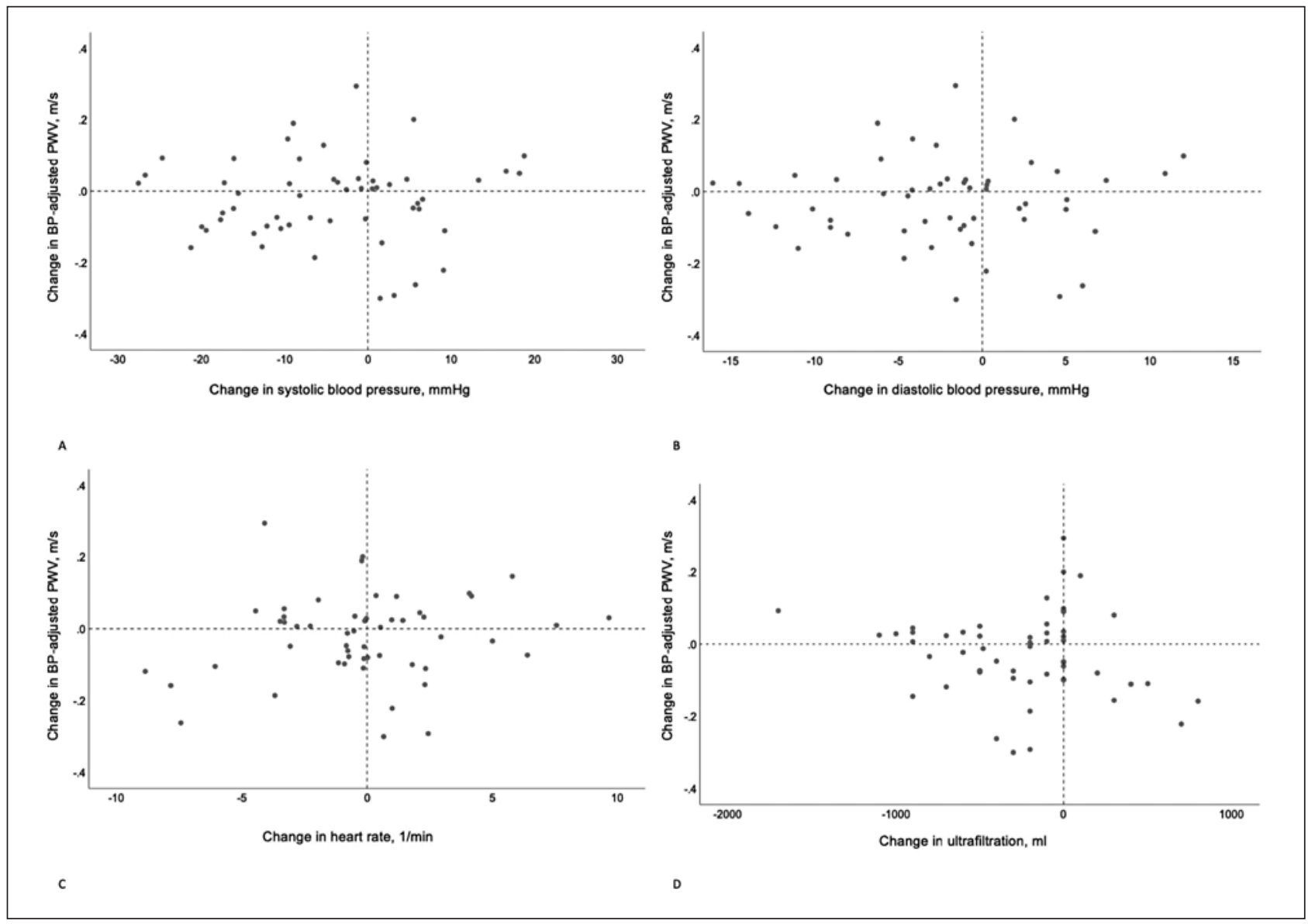

Fig. 3. Change in BP-adjusted PWV related to change in (A) systolic BP; (B) diastolic BP; (C) heart rate; and (D) ultrafiltration between the dialysis sessions during 1 week. BP, blood pressure; PWV, pulse wave velocity. 
Table 4. Comparison of hemodynamically relevant parameters between 3 dialysis days of the week

\begin{tabular}{lcccc}
\hline & Day 1 & Day 2 & Day 3 & $\begin{array}{l}p \text { value, } \\
\text { Friedman-test }\end{array}$ \\
\hline Systolic BP, mean (SD), mm Hg & $125.0(17.6)$ & $120.2(14.0)$ & $119.1(15.0)$ & $0.005^{*}$ \\
Diastolic BP, mean (SD), mm Hg & $72.8(12.1)$ & $69.9(10.4)$ & $68.8(10.0)$ & $<0.001^{\#}$ \\
Pulse pressure, mean (SD), mm Hg & $52.2(11.3)$ & $48.9(42.5-55.3)$ & $50.3(10.1)$ & 0.486 \\
Mean arterial pressure, mean (SD), mm Hg & $96.7(13.8)$ & $93.0(11.2)$ & $91.9(11.5)$ & $0.001^{*}$ \\
Heart rate, b/min & $63.2(56.8-69.7)$ & $64.1(58.7-69.6)$ & $65.7(59.7-71.1)$ & 0.595 \\
Central systolic BP, mean (SD), mm Hg & $111.1(15.5)$ & $107.4(12.7)$ & $107.1(12.8)$ & $0.014^{*}$ \\
Central diastolic BP, mean (SD), mm Hg & $74.1(12.1)$ & $69.9(64.1-75.8)$ & $70.1(10.1)$ & $<0.001^{\#}$ \\
PWV, mean (SD), m/s & $10.7(2.4)$ & $10.5(2.5)$ & $10.5(2.5)$ & $0.003^{*}$ \\
PWVslope, mean (SD), m/s × mm Hg & $0.0350(0.0050)$ & $0.0348(0.0053)$ & $0.0355(0.0045)$ & 0.946 \\
PWVbaseline, m/s & $7.2(5.1-9.3)$ & $6.8(2.6)$ & $6.7(2.6)$ & 0.786 \\
PWV120, mean (SD), m/s & $11.0(2.6)$ & $10.9(2.6)$ & $10.9(2.6)$ & 0.703 \\
Ultrafiltration volume, mean (SD), mL & $2,819(1,021)$ & $2,545(1,102)$ & $2,511(1,115)$ & $0.002^{\#}$ \\
\hline
\end{tabular}

* Statistically significant difference days 1-3.

\# Statistically significant difference days 1-2 and days 1-3.

PWV120, pulse wave velocity adjusted to the level of $120 \mathrm{~mm} \mathrm{Hg}$ central systolic BP and calculated as PWVbaseline +120 $\mathrm{mm} \mathrm{Hg} \times \mathrm{PWVslope}$; normally distributed parameter reported as mean and SD; nonnormally distributed parameter reported as median and interquartile range.

BP, blood pressure; PWV, pulse wave velocity; PWV120, PWV adjusted to $120 \mathrm{~mm} \mathrm{Hg}$ central systolic BP.

Table 5. Independent statistically significant impact parameters on PWV, PWVbaseline, and PWVslope based on multivariable linear regression analysis

\begin{tabular}{llll}
\hline & PWV & PWVbaseline & PWVslope \\
\hline Independent parameters & Systolic BP & Systolic BP* & Systolic BP* \\
& Diastolic BP & Diastolic BP* & \\
& Age & Age $^{\$}$ & \\
& Residual diuresis & Residual diuresis $^{\#}$ & \\
& Loop diuretic & & \\
& PAD $^{\#}$ & & \\
& & & \\
& & & \\
\hline
\end{tabular}

PWV, pulse wave velocity; BP, blood pressure; PAD, peripheral artery disease. ${ }^{*} p<0.05 .{ }^{\#} p<0.01 .{ }^{\$} p<$ 0.001 .

namically relevant parameters between 3 dialysis days of the week. Table 5 shows independent statistically significant influence parameters on PWV, PWVbaseline, and PWVslope based on the results of multiple linear regression analysis.

\section{Discussion}

In this study, we observed no change in PWV120 between dialysis sessions within 1 week in stable outpatients of the single center dialysis unit. PWV and BP parameters varied significantly between the dialysis sessions. The change in BP-adjusted PWV could not be predicted by any parameter, which varied between the dialysis sessions. Though, wide variation ranges were observed for systolic and diastolic BP, heart rate, and ultrafiltration amount between 


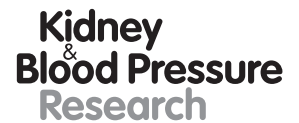

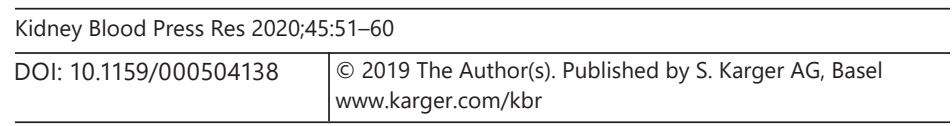

Reshetnik et al.: BP Dependence of AS in ESRD

the dialysis sessions. Our mathematical approach allows the adjustment of the PWV to a particular BP-level (in this study $120 \mathrm{~mm} \mathrm{Hg}$ central systolic BP) and as a consequence possible BP-independent reflection of the true AS as an indicator of the arterial wall damage. Our findings support the expected presumption that the arterial wall structure does not change during 1 week in dialysis patients. The supposed changes in AS are based on the physiologic PWV change due to changes in BP [7]. This conclusion is of direct importance for the interpretation of the studies using PWV either as a cardiovascular risk marker or a PWV change as a therapeutic target. For example, a recently published study reported an association between volume overload and increased AS in patients with moderate to severe CKD [12]. However, volume overload usually causes an increase in BP (also observed in the study). An increased BP can cause increase in AS without any true changes in arterial wall structure. The authors of the study suggested an existing relationship between volume expansion and AS in the discussion. However, this link is confounded by the occurred change in BP.

Two main approaches exist in the literature to convert PWV in a BP-independent parameter: (1) generation of a dimensionless variable as implemented in cardio-ankle vascular index [13] and (2) general adjustment of the PWV values to BP performed on the statistical basis usually employing regression analysis techniques. While cardio-ankle vascular index is not as much substantiated with evidence as conventional aortic PWV, general adjustment of the PWV to BP is not individualized and applicable for study populations or for single subjects [14]. Moreover, the comparability of the values between different studies or during follow-up is difficult. To the best of our knowledge, no studies using individual BP-adjustment of the PWV in dialysis patients have been published so far. Di Iorio et al. [15] studied changes in BP-adjusted PWV in 20 dialysis patients in 1 week. The authors used normalization of the PWV as a method for BP-adjustment - corresponding to a general BP-adjustment approach [15] - and reported no significant change in normalized PWV during 1 week. Noteworthy, carotid-femoral PWV- and BP-measurements have been performed with different devices and the time point of BP- and carotid-femoral PWV-measurement has not been specified further. In case the measurements were not performed simultaneously, the direct link between the particular BP level and measured PWV would be difficult, as the BP varies continuously. Thus, compared to the Di Iorio et al. [15] work our methodological (oscillometry based) approach is possibly more accurate as the method delivers PWV and BP simultaneously. Overall, our data support their findings and extend the evidence that $\mathrm{BP}$-adjustment is necessary to differentiate between physiologic reaction of PWV to a change in BP and true PWV as a reflection of arterial wall damage.

Considering the separate variables in the linear equation created from the point clouds, we defined the line interception point with the Y-axis as a PWVbaseline. This is a virtual parameter because the precondition for interception of the Y-axis would be the $\mathrm{BP}$ of $0 \mathrm{~mm} \mathrm{Hg}$, which is, of course, impossible in real life. However, this parameter seems to reflect the baseline status of the arterial wall as a static component. In our study, PWVbaseline was predicted by systolic and diastolic BP as well as patient age. All factors are known to be the main affecting factors of the AS [6]. Another variable in the linear equation - PWVslope - reflected the intensity of PWV change due to change in BP. This parameter would probably represent the dynamic component of AS or "true" stiffness as is described by the key physical principles [16]. Only systolic BP was able to predict PWVslope in multiple regression analysis of our study collective. Both PWVbaseline and PWVslope might depict different components of AS, which is a reflection of histopathological changes in arterial wall structure such as deterioration of elastin network, vascular smooth muscle hypertrophy, and transformation and increased collagen deposition [17-19]. iPTH was also reported as a main determinant of AS progression [20]. Though the impact of iPTH was significant in the univariate test, in the multivariate analysis, iPTH lost its statistical significance as an independent impact factor on PWV120. 
In conclusion, our data suggest that true AS does not change in the short-term course in dialysis patients and observed changes in PWV are rather associated with BP change due to the intrinsic pressure-dependence. Since PWV has been an acknowledged predictive parameter for cardiovascular risk in non-CKD [21] and CKD population [22], it is essential to determine true changes in PWV and exclude the pressure-dependence component. Our analytical approach represents a novel method for this purpose, which is easy in performance and applicable for large interventional trials and clinical practice. Further studies should be performed to establish the link between BP-adjusted PWV and cardiovascular outcomes.

Though the study has its strengths, we acknowledge several limitations: Due to the device used, the aortic PWV was calculated and not measured. We have previously shown that calculated PWV measured by the Mobil-O-Graph is in a very good agreement with direct measurement of PWV [9]. The observational design of the study allows the description of associations but preclude any causal relationships. Single-center approach resulted in a given participants number. However, this is the largest study describing PB-adjuster PWV individually in dialysis patients so far. Further studies should be conducted to examine the validity of BP-adjusted PWV in other collectives.

\section{Acknowledgments}

Mobil-O-Graphs were kindly provided by the manufacturer.

\section{Statement of Ethics}

The research was conducted in accordance with the World Medical Association Declaration of Helsinki and comply with the guidelines for human studies.

\section{Disclosure Statement}

M.G. has consultancy agreement with IEM (manufacturer of Mobil-O-Graph). There is no conflict of interest for the other authors.

\section{Funding Sources}

No funding source received for this study.

\section{Author Contributions}

A.R.: conception of design, analysis and interpretation of the data, drafting the article, final approval of the version to be published. D.W.: conception of design, analysis and interpretation of the data, drafting the article. G.W.: analysis and interpretation of the data. M.T. and K.-U.E.: providing intellectual content of critical importance. M.G.: interpretation of the data, revising the article, final approval of the version to be published. 


\section{References}

1 Zieman SJ, Melenovsky V, Kass DA. Mechanisms, pathophysiology, and therapy of arterial stiffness. Arterioscler Thromb Vasc Biol. 2005 May;25(5):932-43.

2 Ben-Shlomo Y, Spears M, Boustred C, May M, Anderson SG, Benjamin EJ, et al. Aortic pulse wave velocity improves cardiovascular event prediction: an individual participant meta-analysis of prospective observational data from 17,635 subjects. J Am Coll Cardiol. 2014 Feb;63(7):636-46.

3 Cardoso CR, Salles GF. Aortic Stiffness as a Surrogate Endpoint to Micro- and Macrovascular Complications in Patients with Type 2 Diabetes. Int J Mol Sci. 2016 Dec;17(12):1-12.

4 Lioufas N, Hawley CM, Cameron JD, Toussaint ND. Chronic Kidney Disease and Pulse Wave Velocity: A Narrative Review. Int J Hypertens. 2019 Feb;2019:9189362.

5 Di Lullo L, House A, Gorini A, Santoboni A, Russo D, Ronco C. Chronic kidney disease and cardiovascular complications. Heart Fail Rev. 2015 May;20(3):259-72.

6 Avolio A, Butlin M. Dependence of arterial stiffness on pressure quantified in the realm of the cardiac cycle: towards a patient-specific approach? J Hypertens. 2015 Feb;33(2):257-9.

7 Gavish B, Izzo JL Jr. Arterial Stiffness: Going a Step Beyond. Am J Hypertens. 2016 Nov;29(11):1223-33.

8 Spronck B, Heusinkveld MH, Vanmolkot FH, Roodt JO, Hermeling E, Delhaas T, et al. Pressure-dependence of arterial stiffness: potential clinical implications. J Hypertens. 2015 Feb;33(2):330-8.

9 Reshetnik A, Gohlisch C, Tölle M, Zidek W, Van Der Giet M. Oscillometric assessment of arterial stiffness in everyday clinical practice. Hypertens Res. 2017 Feb;40(2):140-5.

10 Weber T, Wassertheurer S, Hametner B, Parragh S, Eber B. Noninvasive methods to assess pulse wave velocity: comparison with the invasive gold standard and relationship with organ damage. J Hypertens. 2015 May; 33(5):1023-31.

11 Wassertheurer S, Kropf J, Weber T, van der Giet M, Baulmann J, Ammer M, et al. A new oscillometric method for pulse wave analysis: comparison with a common tonometric method. J Hum Hypertens. 2010 Aug;24(8): 498-504.

12 Braam B, Lai CF, Abinader J, Bello AK. Extracellular fluid volume expansion, arterial stiffness and uncontrolled hypertension in patients with chronic kidney disease. Nephrol Dial Transplant. 2019, Epub ahead of print.

13 Shirai K, Utino J, Otsuka K, Takata M. A novel blood pressure-independent arterial wall stiffness parameter; cardio-ankle vascular index (CAVI). J Atheroscler Thromb. 2006 Apr;13(2):101-7.

14 Weber T, Parragh S, Wassertheurer S. Is Blood Pressure Independent Arterial Destiffening Possible? Am J Hypertens. 2017 May;30(5):470-2.

15 Di Iorio B, Nazzaro P, Cucciniello E, Bellizzi V. Influence of haemodialysis on variability of pulse wave velocity in chronic haemodialysis patients. Nephrol Dial Transplant. 2010 May;25(5):1579-83.

16 Chirinos JA. Arterial stiffness: basic concepts and measurement techniques. J Cardiovasc Transl Res. 2012 Jun; 5(3):243-55.

17 Bank AJ, Wang H, Holte JE, Mullen K, Shammas R, Kubo SH. Contribution of collagen, elastin, and smooth muscle to in vivo human brachial artery wall stress and elastic modulus. Circulation. 1996 Dec;94(12):326370.

18 Avolio A, Jones D, Tafazzoli-Shadpour M. Quantification of alterations in structure and function of elastin in the arterial media. Hypertension. 1998 Jul;32(1):170-5.

19 Tölle M, Reshetnik A, Schuchardt M, Höhne M, van der Giet M. Arteriosclerosis and vascular calcification: causes, clinical assessment and therapy. Eur J Clin Invest. 2015 Sep;45(9):976-85.

20 Jung JY, Hwang YH, Lee SW, Lee H, Kim DK, Kim S, et al. Factors associated with aortic stiffness and its change over time in peritoneal dialysis patients. Nephrol Dial Transplant. 2010 Dec;25(12):4041-8.

21 Williams B, Mancia G, Spiering W, Agabiti Rosei E, Azizi M, Burnier M, et al.; ESC Scientific Document Group. 2018 ESC/ESH Guidelines for the management of arterial hypertension. Eur Heart J. 2018 Sep;39(33):3021104.

22 Townsend RR, Anderson AH, Chirinos JA, Feldman HI, Grunwald JE, Nessel L, et al.; CRIC Study Investigators. Association of Pulse Wave Velocity With Chronic Kidney Disease Progression and Mortality: Findings From the CRIC Study (Chronic Renal Insufficiency Cohort). Hypertension. 2018 Jun;71(6):1101-7. 